\title{
BMJ Open What information needs do people with recently diagnosed diabetes mellitus have and what are the associated factors? A cross-sectional study in Germany
}

\author{
Sandra Grobosch, ${ }^{1,2,3}$ Silke Kuske, ${ }^{1,2}$ Ute Linnenkamp, ${ }^{1,3}$ Nicole Ernstmann, ${ }^{4}$ \\ Astrid Stephan, ${ }^{2}$ Jutta Genz, ${ }^{1}$ Alexander Begun, ${ }^{1,5}$ Burkhard Haastert, ${ }^{2,6}$ \\ Julia Szendroedi, ${ }^{3,7,8}$ Karsten Müssig, ${ }^{3,7,8}$ Volker Burkart, ${ }^{3,8}$ Michael Roden, ${ }^{3,7,8}$ \\ Andrea Icks, ${ }^{1,2,3}$ for the GDS Group
}

To cite: Grobosch S, Kuske S, Linnenkamp U, et al. What information needs do people with recently diagnosed diabetes mellitus have and what are the associated factors? A cross-sectional study in Germany. BMJ Open 2018;8:e017895. doi:10.1136/ bmjopen-2017-017895

- Prepublication history and additional material for this paper are available online. To view these files, please visit the journal online (http://dx.doi. org/10.1136/bmjopen-2017017895).

SG and SK shared first authorship.

MR and Al shared senior authorship.

Received 8 June 2017 Revised 27 July 2018 Accepted 27 September 2018

Check for updates

(C) Author(s) (or their employer(s)) 2018. Re-use permitted under CC BY-NC. No commercial re-use. See rights and permissions. Published by BMJ.

For numbered affiliations see end of article.

Correspondence to Sandra Grobosch; sandra.grobosch@ddz.uniduesseldorf.de

\section{ABSTRACT}

Objectives This study aimed to identify: (1) information needs of people with recently diagnosed type 1 or type 2 diabetes mellitus (DM); (2) information needs within different subgroups; and (3) factors associated with information needs concerning DM such as current level of information, health-related quality of life or participation preferences.

Design A mixed-method approach combining quantitative and qualitative methods was used. Information needs for different topics and estimated associated factors were described using logistic regression models. Additionally, a qualitative content analysis was performed.

Setting Monocentre study.

Participants Information needs were assessed and analysed in 138 consecutive participants with DM who took part in the German Diabetes Study (54\% type 2 diabetes, $64 \%$ male, mean age $46.3 \pm 12.3$ years, known diabetes duration $<1$ year).

Results Most participants displayed a need for information in all topics provided, especially in diabetes research (86\%) and treatment/therapy (80\%). Regarding those topics, participants wished for information regarding new treatments that simplify their everyday life. In general, participants preferred topics that focus on the management or handling of DM over topics related to clinical factors of DM, such as causes and complications. A low current level of information and treatment with antihyperglycaemic medication were significantly associated with higher information needs, and diabetes-related comorbidity and higher mental component summary score in the 36-Item Short-Form Health Survey (SF-36) with lower information needs.

Conclusion People with recently diagnosed DM display high information needs, which differ according to the current level of information, mode of diabetes treatment, diabetes-related comorbidity and mental component summary score in the SF-36. There appears to be a preference for information, which can help to simplify life with diabetes and for information that corresponds to their level of knowledge. This should be considered in patient information activities.
Strengths and limitations of this study

- A strength of the present study is the ability to analyse information needs in people with recently diagnosed diabetes, a relevant patient group for the provision of suitable information.

- A large number of variables and their association with information needs could be analysed.

- A limitation is the cross-sectional design.

- Furthermore, the present observational study was not designed as a population-based study with a representative sample; for example, our cohort included more male and younger participants as well as more highly educated participants.

Trial registration number NCT01055093.

\section{INTRODUCTION}

Diabetes mellitus (DM) is composed of different abnormalities associated with chronic hyperglycaemia and is characterised by complex self-management tasks. ${ }^{1}$ Patients require high-quality and evidencebased information to enable adequate decision making. ${ }^{2}$ People with DM show a higher information need compared with people with other diseases, such as cardiovascular and respiratory diseases. ${ }^{3}$ However, despite existing efforts to improve available information and a growing discussion of associated factors such as health literacy, patients' information needs are frequently disregarded.

A recent systematic review revealed surprisingly few studies addressing the information needs of people with $\mathrm{DM}^{4}$ in particular in people with recently diagnosed diabetes. As of today, there is only one study that analyses 
information needs in people with recently diagnosed diabetes. ${ }^{5}$ However, only people with type 2 diabetes were involved, and only qualitative methods were used. Several questions remain unanswered, such as whether there are differences between patient subgroups and which factors are associated with information needs.

Thus, the present study aims to identify and analyse: (1) information needs of people with recently diagnosed type 1 or type $2 \mathrm{DM}$; (2) information needs within different subgroups; and (3) factors associated with information needs concerning DM such as current level of information, health-related quality of life or participation preferences. An information need is defined as the 'recognition that their knowledge is inadequate to satisfy a goal, within the context/situation that they find themselves at a specific point in the time'. ${ }^{6}$

\section{METHODS}

\section{Study design and population}

The present cross-sectional study combined quantitative and qualitative methods (mixed-methods) using baseline data of participants in the German Diabetes Study (GDS). GDS is an ongoing prospective observational study initiated and coordinated by the German Diabetes Center. ${ }^{7}$ The GDS aims to investigate the course of disease and the consequences of DM and has been described in detail elsewhere. ${ }^{7}$ Participants are people aged between 18 years and 69 years with recently diagnosed DM with a duration of less than 12 months of known diabetes. Data assessment comprises standardised questionnaires and interviews, detailed physical examinations and comprehensive metabolic phenotyping.

The present study included 157 consecutive participants from the GDS between February 2014 and May 2016. Nineteen participants were excluded due to missing variables, yielding 138 for the final analysis.

\section{Ethical approval}

This study is performed according to the Declaration of Helsinki. $^{7}$

\section{Patient and public involvement}

Patients and public were not involved in the present study. The questionnaire for measuring the need for information was developed with the participation of people with DM in focus groups.

\section{Assessment of information needs}

Information needs were assessed using a questionnaire developed and evaluated by Chernyak et $a l^{8}$ (online supplementary appendix 1). The German language version has been previously applied to a clinic-based population of people with DM. ${ }^{8}$ The questionnaire is based on a mixed-methods design, namely a partially mixed concurrent equal status design. ${ }^{9}$ Both quantitative and qualitative data were assessed without prioritising either of the methods.
It includes 11 topics of information needs ${ }^{8}$ : 'causes of diabetes', 'course of the disease', 'treatment/therapy', 'acute complications', 'late complications', 'diabetes in everyday life', 'mental strain', 'lifestyle adjustment, health promotion and prevention', 'support, helplines and information sources', 'social and legal aspects' and 'diabetes research'. Participants are able to mark whether information is currently needed (no=0/yes $=1$ ) and assess their current level of information for each topic (very well, well, not well and not informed at all). Furthermore, participants can prioritise a maximum of three topics for which they currently need information. A blank text field is provided per information need to specify selected needs: 'Please explain what particular interests you have about these topics'. They can also add an individual unlisted information need in any question. At the end of the information needs questionnaire, the participants have the opportunity to reply to the question 'What do you consider to be particularly important with regard to information on diabetes?' in a blank text field.

\section{Variables}

Outcome: category of information need

Three categories of information needs were defined for the purposes of the present study. The first was the desire for information $($ no $=0 /$ yes $=1)$ on diabetes research. The second category focused on topics related to clinical factors of DM including a need for information on the causes of diabetes, course of the disease, acute complications, long-term complications and mental strain. The needs identified in the third category focused on the management and handling of DM including management-related topics, treatment/therapy, diabetes in everyday life, lifestyle adjustment, health promotion and prevention, support, helplines and information sources and social and legal aspects. Within the second and third categories, results were summed up and dichotomised into 'low information needs' (ranging from 0 to 2) or 'high information needs' (ranging from 3 to 5).

\section{Factors associated with information needs}

The associated factors were taken from the data assessed in GDS as described above. The variables were selected as follows: first, a set of variables was deduced empirically from the existing literature for quantitative analysis. ${ }^{10-15}$ Studies showed that age (years), sex, education, type of diabetes, mode of diabetes treatment and health status appear to have an impact on information needs. ${ }^{10-15}$ Education was coded by 'other graduation' and 'university degree'; the type of diabetes was coded by 'type 1', 'type 2' and 'other'; mode of diabetes treatment was coded by 'no antihyperglycaemic medication', 'oral glucose-lowering drugs' and 'insulin'. Health status was defined according to diabetes-related comorbidities (nephropathy, neuropathy, peripheral arterial occlusive disease, myocardial infarction, stroke and transient ischaemic attack). 
Second, five explorative groups of thematically relevant variables in the context of diabetes were developed on a theoretical basis:

i. Socioeconomic factors are associated with diabetes-related information-seeking behaviour. ${ }^{16}$ Further socioeconomic factors in addition to education, which has already been included, were therefore included: employment coded by 'no' or 'yes'; school graduation defined as 'other graduation' and 'graduation from high school'; and migration background, denoted by place of birth other than Germany or nationality other than German.

ii. Past diabetes experience is associated with information needs. ${ }^{4}$ It can therefore be assumed that diabetes-related and health-related factors may have an impact on information needs. Hence, besides diabetes type and mode of diabetes treatment that have already been included, the duration of DM (time since diagnosis until inclusion in the GDS), hemoglobin $\mathrm{A}_{1 \mathrm{c}}\left(\mathrm{HbA}_{1 \mathrm{c}}\right)$ and number of overall drugs were also included.

iii. As some studies on information needs also report on participation preferences and on the people's knowledge, ${ }^{4}$ this variable was added. Self-reported participation preferences, and thus the wish to be involved in medical decision making, were measured by the Control Preference Scale, coded by 'passive role', 'collaborative role' and 'active role'. ${ }^{17}$ The information needs questionnaire included questions about current level of information. The current level of information on diabetes research was coded by 'high current level of information' (very well or well informed) and 'low current level of information' (not well or not informed at all). The other two categories of information needs were summed up and dichotomised into 'high current level of information' (ranging from 0 to 6 ) as well as 'low current level of information' (ranging from 7 to 15).

iv. The fourth group of variables refers to depression and health-related quality of life. People with DM have a higher prevalence of depression and a lower health-related quality of life than people without DM. ${ }^{18} 19$ This may lead to a lower level of activity. Depression was measured using the Center for Epidemiological Studies Depression Scale, long German version (ADS-L $)^{20}$ and Problem Areas in Diabetes (PAID) survey. ${ }^{21}{ }^{22}$ In accordance with the respective published evaluation methods, depression was coded according to ADS-L as 'depressive symptoms' (cut-off score >22) and according to PAID as 'severe diabetes-related distress' (cut-off score $\geq 40$ ). Health-related quality of life was measured using the 36-Item Short-Form Health Survey $(\mathrm{SF}-36)^{23}{ }^{24}$ and analysed according to the physical and mental summary scales. In addition, the 5-Item WHO Well-Being Index questionnaire was analysed, and well-being was coded as 'low well-being' (ranging from 0 to 12) and 'high well-being' (ranging from 13 to 25 ) ${ }^{25}$ v. Several studies have found that 'self-management' and 'lifestyle' are the main contents of the information needs of people with $\mathrm{DM},{ }^{4}$ and thus the present study sought to identify a possible association. Selfmanagement was operationalised using three questions to be answered with yes or no: 'Do you have a health pass for diabetes?', 'Do you perform glucose self-monitoring?' and 'Have you ever participated in an education programme for people with diabetes?'. Variables that provide statements on the participants' lifestyles were included: body mass index (BMI), smoking behaviour and leisure time activity. BMI was categorised in accordance with the WHO definition, ${ }^{26}$ smoking behaviour was coded by 'no answer', 'no' and 'yes'. Leisure time activity was operationalised according to the Baecke index ${ }^{27} 28$ as a summary of the variables: 'During leisure hours, I walk', 'During leisure hours, I ride a bike' and 'For how many minutes a day do you walk or ride a bike going back and forth from work, school or shopping?'.

\section{Quantitative analysis}

First, descriptive summaries were obtained (depending on the distribution of the variables by frequencies, percentages, means \pm SD). Participants' current levels of information were described in percentages. Comparisons between the different categories of information needs were carried out using McNemar's test.

To estimate associations between the information need categories as described above and associated factors, multivariate logistic regression models were fitted, resulting in ORs with 95\% CI corresponding to one unit changes of the independent variable. Three groups of models were fitted, using the categories of information needs (high vs low) as a dependent binary variable.

The following steps were performed to select the final set of independent variables: we first included the six groups of variables described above fitting different models separately. We excluded variables due to many missing values, low impact in the regression analysis, low variation or high correlation to other covariables. Larger models were then fitted, which included the independent variables of all six groups. After discussion of these models, fixed sets of independent variables including confounders were selected for the three main models. The final set of variables included: age, sex, education, mode of diabetes treatment (antihyperglycaemic medication yes vs no), diabetes-related comorbidity (binary), current level of information (high vs low), health-related quality of life (mental and physical component summary score in the SF-36) and BMI $\left(\geq 30 \mathrm{~kg} / \mathrm{m}^{2} \mathrm{vs}<30 \mathrm{~kg} / \mathrm{m}^{2}\right)$.

With regard to the research-related information needs outcome, the corresponding model was only fitted in the subpopulation of subjects with type 2 diabetes, since all participants from the type 1 subgroup were in need of information on diabetes research. The models for the clinical and management-related information needs outcomes were run both for type 1 and type 2 diabetes. 
Table 1 Participants' characteristics

\begin{tabular}{|c|c|c|c|}
\hline Characteristics & & $\mathbf{N}(\%)$ & Mean (SD) \\
\hline Total number of participants & & 138 & \\
\hline Age, $n=138$ & & & $46.3(12.3)$ \\
\hline \multirow[t]{2}{*}{ Sex, $n=138$} & Male & $88(64)$ & \\
\hline & Female & $50(36)$ & \\
\hline University degree, $\mathrm{n}=135$ & & $64(47)$ & \\
\hline Employment, $\mathrm{n}=137$ & & $111(81)$ & \\
\hline Migration background, $n=136$ & & $18(13)$ & \\
\hline \multirow[t]{3}{*}{ Type of diabetes, $n=138$} & Type 1 & $56(41)$ & \\
\hline & Type 2 & $75(54)$ & \\
\hline & Other & $7(5)$ & \\
\hline \multirow[t]{4}{*}{ Mode of diabetes treatment, $n=130$} & No antihyperglycaemic medication & $26(20)$ & \\
\hline & Oral glucose-lowering drugs & $51(39)$ & \\
\hline & Insulin & $50(38)$ & \\
\hline & Oral glucose-lowering drugs and insulin & $3(2)$ & \\
\hline Number of overall drugs, $n=130$ & & & $2.98(1.9)$ \\
\hline Diabetes-related comorbidity, $n=136$ & & $23(17)$ & \\
\hline
\end{tabular}

The mode of diabetes treatment was excluded for type 1 diabetes because only one participant in that subgroup did not use antihyperglycaemic medication. The data analysis for this paper was generated using SAS software, V.9.4.

\section{Qualitative analysis}

The qualitative content analysis was used for the free-text entries and performed according to Elo and Kyngäs. ${ }^{29}$ A coding tree was developed by two coders, and one coder analysed all entries and the other reviewed the coding. According to the questionnaire, the theoretical and deductive predefined information need categories were first analysed deductively. A subsequent inductive analysis was performed to determine the subcategories. The inductive analysis entailed 'open coding, creating categories and abstraction'. During that phase, the data were abstracted and described in order to define higher-order categories.

\section{RESULTS}

\section{Participant characteristics}

Approximately $60 \%$ of the participants were male (table 1). About half of them had a university degree, and about three quarters were employed. One in eight had a migration background. More than $50 \%$ had type 2 diabetes, and about one-fifth were treated without antihyperglycaemic medication. Participants took an average of three different drugs. Diabetes-related comorbidity was present in every sixth person.

\section{Current level of information}

Most participants were not well informed or not informed at all about diabetes research (67.9\%) (figure 1).
Regarding clinical topics, the majority of participants reported that they were very well or well informed about causes of diabetes $(69.1 \%)$, long-term complications $(68.7 \%)$, course of the disease $(66.7 \%)$ and acute complications $(60 \%)$. Mental strain $(63.9 \%)$ was the only topic where not well informed or not informed at all constituted the majority. The majority of participants reported that they were very well or well informed about the following management-related topics: treatment/therapy $(76.9 \%)$, diabetes in everyday life $(64.4 \%)$, and lifestyle adjustment, health promotion and prevention (58.5\%). The majority of participants stated that they were not well informed or not informed at all regarding the topics support, helplines and information sources $(56.7 \%)$ and social and legal aspects $(74.1 \%)$. There were more participants with a high current level of information on clinical topics $(48.1 \%)$ than with a high current level of information on management-related topics $(35.6 \%)$ (McNemar's test: $\mathrm{p}=0.007$ ).

\section{Quantitative results Information needs}

When asked which topics they would like information on, the majority of participants stated a need for information on all topics listed in the questionnaire (figure 2). Most of them $(85.8 \%$ ) wished to have more information about diabetes research. Of the clinical topics, participants showed the greatest need for information on the course of the disease $(66.1 \%)$. The lowest need was stated for information on acute complications $(60.3 \%)$ and mental strain $(56.6 \%)$. Management-related topics, for example, treatment/therapy $(80.5 \%)$ and lifestyle adjustment, and health promotion and prevention $(77.9 \%)$ were generally of more interest than clinical topics. The lowest 


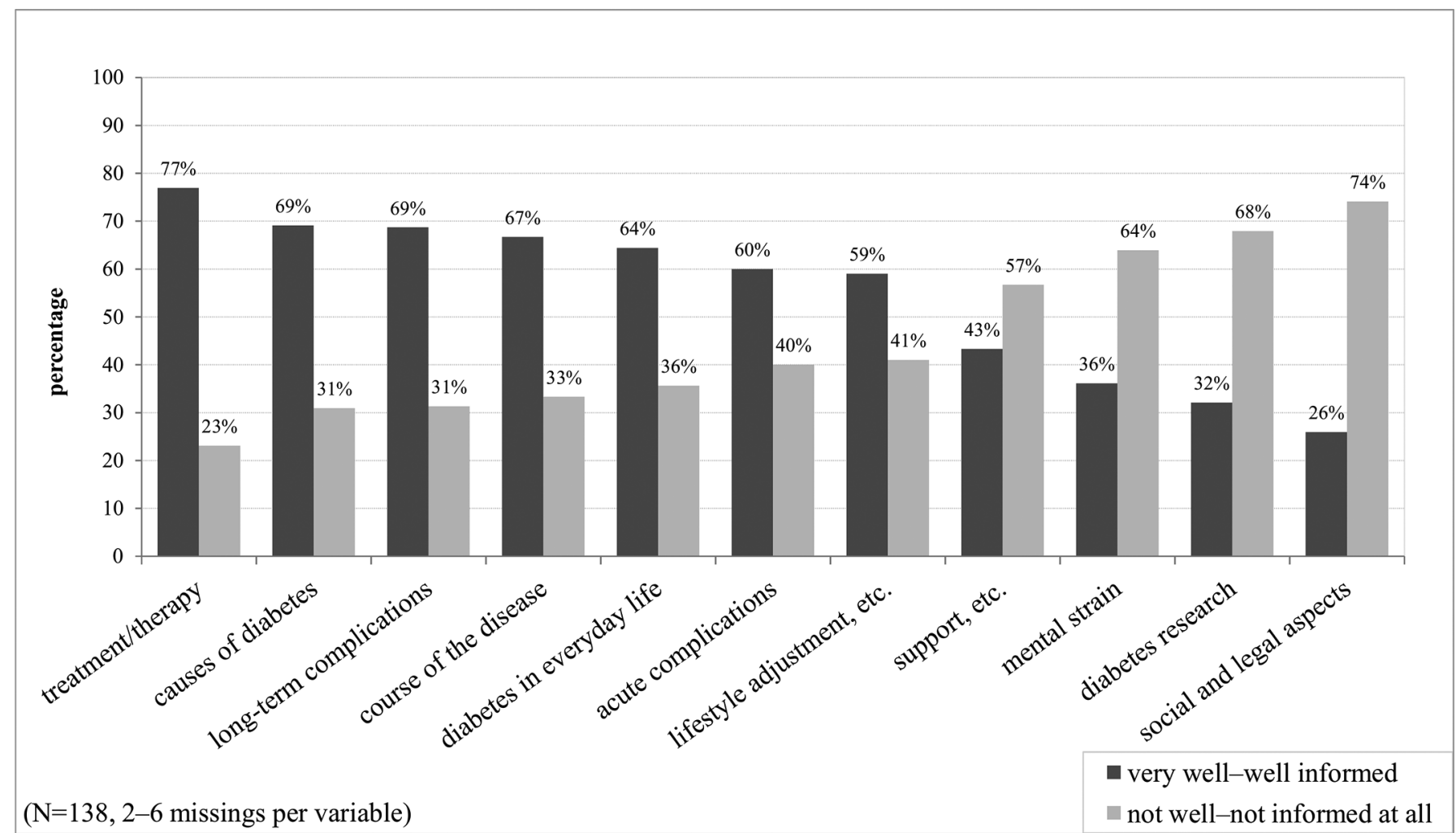

Figure 1 Current level of information of the study population on the diabetes-related topics.

information need for management-related topics was found for support, helplines and information sources $(62.9 \%)$. Four participants stated no information need.
One hundred and sixteen participants selected three prioritised topics, while some participants selected only two $(n=10)$ or one $(n=5)$. Figure 3 shows the percentage

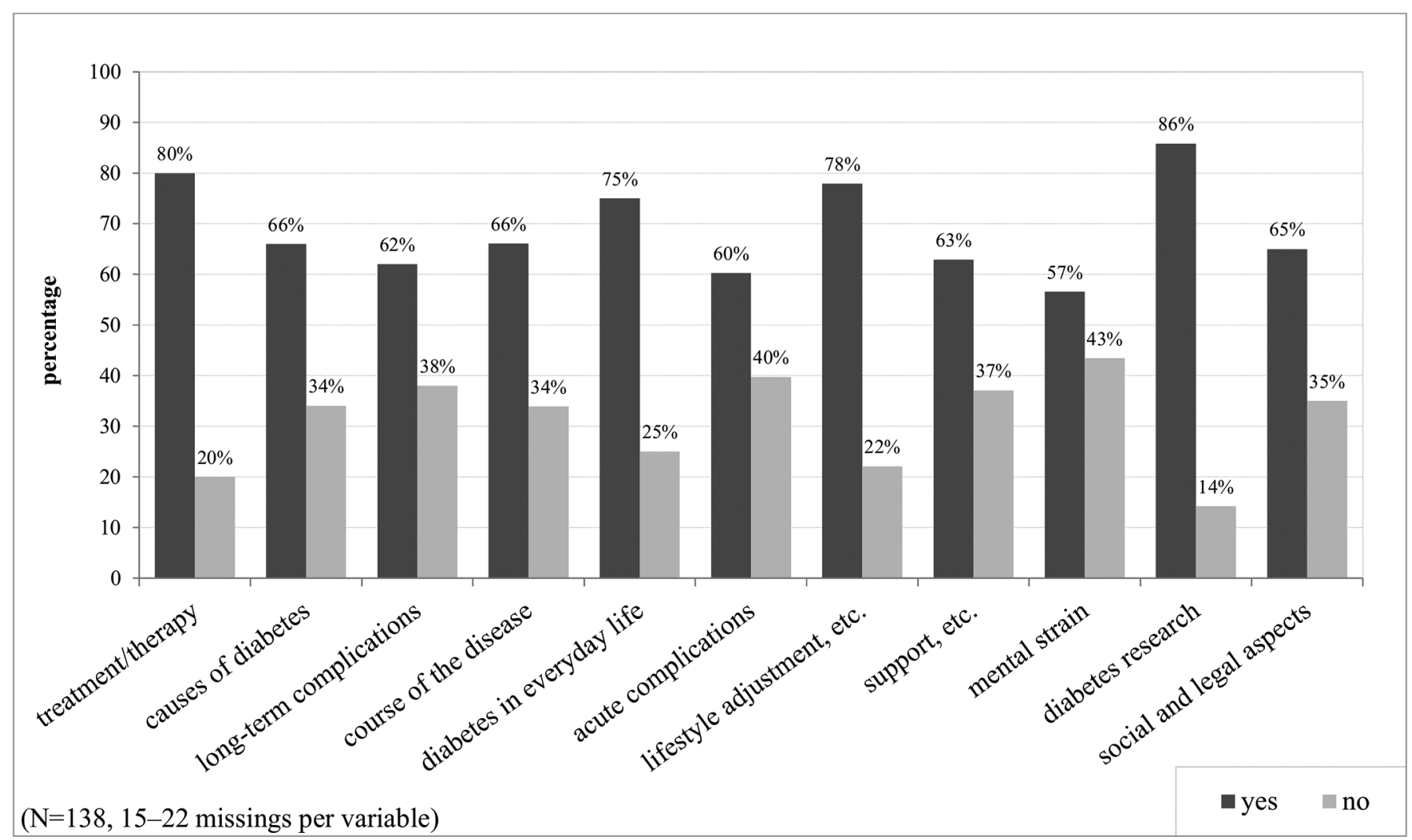

Figure 2 Information needs of the study population. 


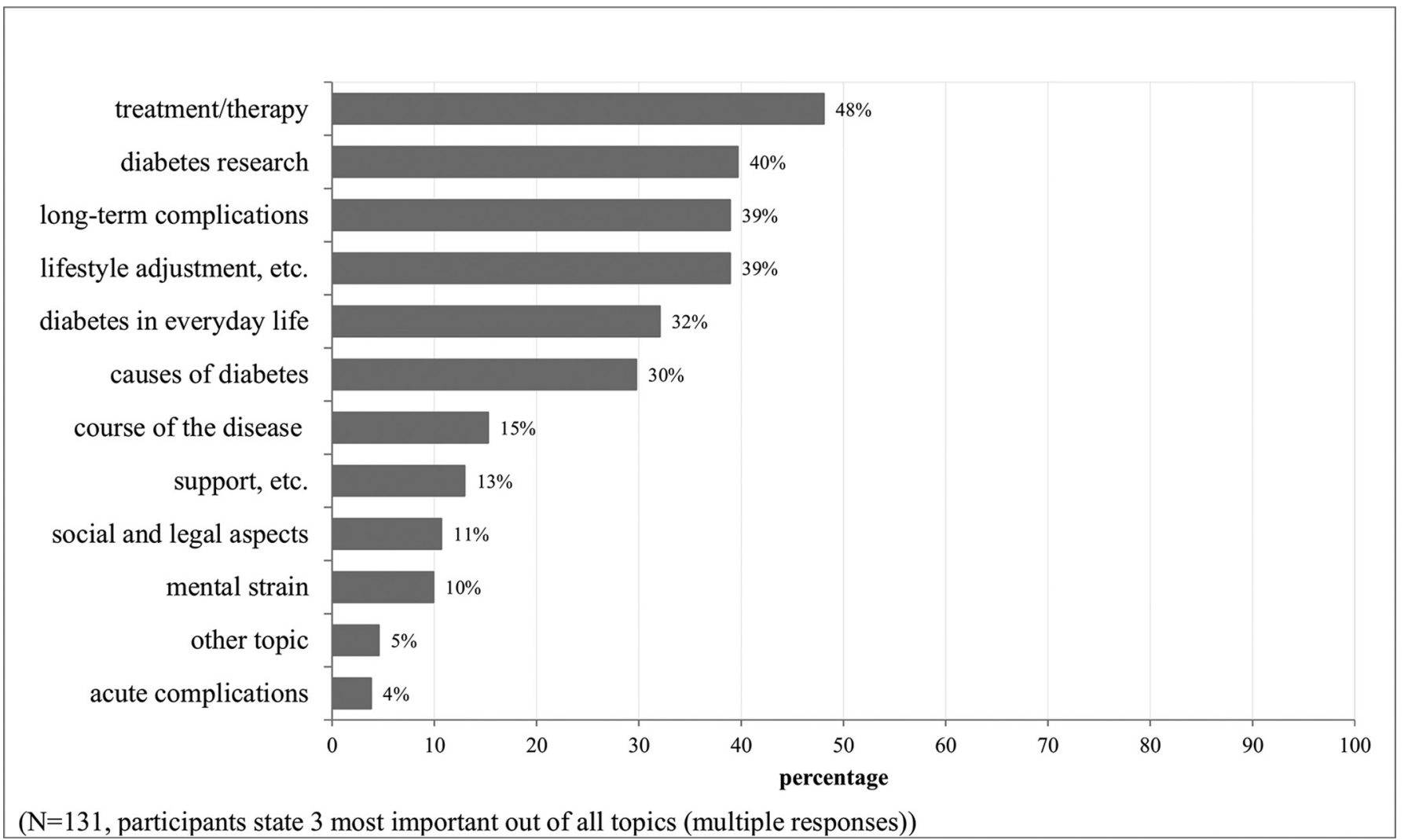

Figure 3 Topics mentioned as most important by participants.

with which each topic was selected as the priority from all possible options (relative to all 131 participants with valid data). When asked to rank the three most important topics (page one of the questionnaire, online supplementary appendix 1), participants prioritised information about diabetes research $(39.7 \%)$ more than most topics allocated to the other two categories. A high information need was also reported for the clinical topics long-term complications $(38.9 \%)$ and causes of diabetes $(29.8 \%)$. The topics course of the disease $(15.3 \%)$ and mental strain $(9.9 \%)$, and especially the topic acute complications $(3.8 \%)$, were rarely prioritised. The highest priority was reported for information about treatment/therapy as a management-related topic $(48.1 \%)$. In the category management-related topics, high information needs were also reported for lifestyle adjustment, health promotion and prevention $(38.9 \%)$ and diabetes in everyday life $(32.1 \%)$. The topics support, helplines and information sources $(13 \%)$ and social and legal aspects $(10.7 \%)$ were rarely prioritised.

\section{Associated factors}

The multiple logistic regression models for participants with type 1 diabetes (online supplementary appendix 2a) showed that the current level of information in clinical and management-related topics is significantly associated with information needs (OR 0.17 (0.03-0.92) and $0.11(0.02-0.75))$. In people with type 1 diabetes, a higher mental component summary score in the
SF-36 is significantly associated with low information needs concerning management-related topics (OR 0.87 (0.76-0.995)).

Participants with type 2 diabetes (online supplementary appendix 2b) treated with antihyperglycaemic medication were more likely to have information needs regarding diabetes research compared with those without antihyperglycaemic medication (OR 6.98 (1.38-35.21)). Existing comorbidities in people with type 2 diabetes were associated with low information needs regarding diabetes research (OR $0.04(0.01-0.38)$ ). However, low statistical power should be considered in the interpretation of the non-significant results.

If a Bonferroni adjustment for multiple testing for the number of independent variables were to be considered, only the association of need for diabetes research and diabetes-related comorbidity would remain significant in subjects with type 2 diabetes.

\section{Qualitative results}

Qualitative analysis showed that participants who sought information about topics in the category diabetes research specifically expressed a need for information on study participation and results, scientific developments (especially for cures, treatment (eg, artificial pancreas)) and technical devices (eg, blood glucose measurement).

Specific information needs that were stated for clinical topics, such as causes of diabetes, were: causes of latent autoimmune diabetes in adults and people with type 1 
diabetes in older age. Participants wanted to know more about the course of the disease, especially a description of the disease process and positive influences on the course of the disease. Wishes for information about acute complications were not explained in more detail. As far as long-term complications are concerned, participants expressed specific needs for information regarding the conditions under which these long-term complications occur and how symptoms can be prevented and recognised. Needs for information regarding mental strain included information on the impact on daily life, stress management and fear of hypoglycaemia.

Participants who were interested in the management-related topics category expressed specific information needs about treatment/therapy, in particular information on existing and new treatment options (eg, continuous glucose monitoring and insulin pump therapy) and information about simplified therapy, especially with less measuring and fewer insulin syringes. Specific needs in diabetes in everyday life were: coping strategies in certain situations including tips for simplification (eg, holidays and work), diabetes management (eg, time management, calculating insulin or bread units) and interaction with people with DM. Information needs in the lifestyle adjustment, health promotion and prevention category included information about sports and nutrition, tips and strategies for handling diabetes better and possibilities to share experiences (eg, health insurance and weight-loss clinic). In the support, helplines and information sources category, participants expressed interest in an overview of existing support offers and education programmes. Participants who prioritised social and legal aspects wanted information about diabetes as a disability and job-related information (eg, terminating employment).

The results of the last open question identified a preference for information to be provided personally, in brochure and video form, or at specific information events. Patients expressed a preference for information to be provided at all times especially recently after diagnosis and when new insights are gained and for it to be comprehensive, transparent, neutral and of high quality. Furthermore, participants expressed a wish for information to be adapted to their level of knowledge.

\section{Synthesis of quantitative and qualitative results}

The greatest level of interest was shown in the two categories diabetes research and management-related topics, particularly the topic treatment/therapy in the latter. Where diabetes research is concerned, participants requested more information on new treatments and technical devices. In both topics, there was a strong desire for information about new insights to simplify treatment. Simplification and disease management are core qualitative aspects that appear to be relevant to coping strategies in daily live. Individual characteristics such as existing knowledge appear to be particularly relevant to information needs and information provision. It can also be noted that participants requested information to be adapted to their level of knowledge.

\section{DISCUSSION}

Participants with recently diagnosed DM have a high information need in all the topics concerning diabetes that were assessed with the information needs questionnaire. They express a particular need for diabetes research and prefer more management-related topics than clinical topics. Information needs concerning DM seem to be associated with current level of information, mode of diabetes treatment, diabetes-related comorbidity and mental component summary score in the SF-36.

The highest information need concerned diabetes research. This may be due to the fact that participants in the GDS are more interested in research questions than people with DM who do not participate in a research study. ${ }^{730}$ The qualitative results indicate that participants wish information to be up to date with the latest scientific findings. Another aim could be to verify information provided by their physician. ${ }^{31}$ Other studies have also identified an interest in information on recent scientific development. ${ }^{4}$

In general, participants requested more information on management-related topics than on clinical topics. The qualitative data clearly show that the explanation of clinical topics frequently includes management-related information. For example, participants stated that they would like to receive more information on stress management. Resource-oriented provision of information is therefore more likely to meet the needs of people with recently diagnosed diabetes. It can be assumed that this is related to the stage at which the recent diagnosis of diabetes was made and a presumably better health status. A high need for information about treatment/therapy has also been identified by other studies. ${ }^{512} 1431-34$

In people with type 1 diabetes, the analysis of the two categories clinical topics and management-related topics showed that a low current level of information is associated with a higher need for information. However, despite being currently well informed, participants still required information on treatment/therapy. An explanation could be: although people feel well informed, they do not have the specific information that helps them to achieve their personal goal (for instance the simplification of everyday life). The qualitative data show that a number of participants would like more detailed information that is adapted to their level of knowledge. In contrast, information on mental strain was rarely prioritised, although a low current level of information was reported. St. Jean posited that a lack of information sources or unconscious information could account for why relevant information cannot be obtained. ${ }^{31}$ The low information need concerning mental strain may also be explained by the fact that the recently diagnosed participants do not experience mental strain.

A higher mental component summary score in the SF-36 was associated with lower information needs in management-related topics in people with type 1 diabetes. The health-related quality of life of people with type 1 diabetes is often reduced because of diabetes-related factors, for 
example, fear of hypoglycaemia (also reported as an information need in the qualitative results). ${ }^{35}$ In this study, people with a higher mental component summary score in the SF-36 may feel that they do not need any further information to manage their situation. Other studies show that optimistic feelings and support in diabetes experience were associated with different information needs in people with DM. ${ }^{4}$

In people with type 2 diabetes, antihyperglycaemic medication appears to be associated with a greater need for information on diabetes research. This finding confirms a focus group analysis by Lamberts et al that showed a greater need for drug-related information in people who have recently started treatment with oral glucose-lowering drugs. ${ }^{14}$

Surprisingly, diabetes-related comorbidity in people with type 2 diabetes was associated with a lower need for information for diabetes research. No other study reported this association. Adjustments were made for the current level of information, but it cannot be ruled out that people with diabetes-related comorbidities are already well informed.

No associations were found between information needs and sex, age or further variables, possibly due to an insufficient statistical power to detect further significant associations.

Regarding the clinical implications of this study, results may contribute to an adjustment of the design of communication strategies and education programmes at an early stage of the disease. Some people with DM felt that they received enough information about diabetes and therefore did not attend self-management education programmes. ${ }^{36}$ An individual and patient-centred approach to building programmes can increase participation.

\section{Limitations and strengths}

The present observational study was not designed as a population-based study and therefore does not claim to represent the entire German diabetes population. Rather, it seeks to reveal predictors associated with later outcomes (eg, diabetes-associated cardiovascular complications) in specific subgroups and to unravel underlying mechanisms. ${ }^{37}$ Compared with population-based representative samples, our cohort included more male and younger participants as well as more highly educated participants. Nevertheless, anthropometric data, such as BMI, were comparable with other German or European cohorts. ${ }^{37}$ However, the selection may introduce bias because the patients who participated in the GDS were potentially more motivated, which could suggest a higher current level of information.

A limitation of the present study is its relatively low sample size and the large number of variables to be investigated as possible risk factors and confounders for information need. There is low statistical power to detect weaker associations. The results should therefore be interpreted with caution. In the 'final models', associations might be overestimated because of data-driven selection. However, due to the low sample size, it was not possible to separate the data into two sets of training and test data for model building and validation of the final model. Furthermore, because of multiple testing in many different regression models, some significant results might have occurred by chance with respect to alpha inflation. Reference is made to the effect of a possible Bonferroni adjustment in the results section.

The strengths of the present study are the possibility to analyse information needs in people with recently diagnosed diabetes, a relevant patient group for the provision of suitable information. It is noted that information needs may rise with the progression of the disease. ${ }^{31}$ The longitudinal design of GDS will allow a prospective analysis of the patients in this study. Another strength is: a large number of variables and their association with information needs could be analysed.

\section{CONCLUSION}

In people with recently diagnosed diabetes, there is currently a high information need for all topics concerning diabetes, especially diabetes research and management-related topics, although study participants reported a relatively high level of being informed. Participants expressed a particular need for information regarding simplification of life with diabetes and for information adapted to their level of knowledge. Information needs differ between patient groups in that information needs are associated with the current level of information, mode of diabetes treatment, diabetes-related comorbidity and mental component summary score in the SF-36. This has to be considered when patients are provided with information about their disease. An open question is how information needs might change over the course of the disease. The prospective GDS provides the opportunity to analyse this question in the future.

\section{Author affiliations}

${ }^{1}$ Institute for Health Services Research and Health Economics, German Diabetes Center, Leibniz Center for Diabetes Research at the Heinrich Heine University Düsseldorf, Düsseldorf, Germany

${ }^{2}$ Institute for Health Services Research and Health Economics, Centre for Health and Society, Faculty of Medicine, Heinrich Heine University Düsseldorf, Düsseldorf, Germany

${ }^{3}$ German Center for Diabetes Research (DZD), München-Neuherberg, Germany ${ }^{4}$ Department for Psychosomatic Medicine and Psychotherapy, Center for Health Communication and Health Services Research, University Hospital of Bonn, Bonn,

Germany

${ }^{5}$ Institute for Biometrics and Epidemiology, German Diabetes Center, Leibniz Center for Diabetes Research at the Heinrich Heine University Düsseldorf, Düsseldorf, Germany

${ }^{6}$ mediStatistica, Neuenrade, Germany

${ }^{7}$ Division of Endocrinology and Diabetology, Faculty of Medicine, Heinrich Heine University Düsseldorf, Düsseldorf, Germany

${ }^{8}$ Institute for Clinical Diabetology, German Diabetes Center, Leibniz Center for Diabetes Research at the Heinrich Heine University Düsseldorf, Düsseldorf, Germany 
Collaborators The GDS Group consists of M Roden (speaker), H Al-Hasani, A E Buyken, J Eckel, G Geerling, C Herder, A Icks, J Kotzka, 0 Kuß, E Lammert, D Markgraf, K Müssig, W Rathmann, J Szendroedi, D Ziegler and their coworkers.

Contributors Al, MR, JS, KM, VB, SG, SK, UL, NE, AS and JG contributed to the concept, design and drafting of the present study. Al, SG, SK, AB and BH developed the design of the analysis. SG, AB and BH conducted formal analysis. SK and Al supervised the analysis process. SG, SK and Al contributed to the writing of the manuscript; all authors were involved in editing. All authors read and approved the final manuscript.

Funding The present study was funded by the German Federal Ministry of Education and Research (BMBF) to the German Center for Diabetes Research (DZD e.V.) and the Research Commission of the Faculty of Medicine of the Heinrich Heine University Düsseldorf. The German Diabetes Study was initiated and financed by the DDZ - German Diabetes Center, which is funded by the German Federal Ministry of Health (Berlin, Germany) and the Ministry of Culture and Science of the state North Rhine-Westphalia (Düsseldorf, Germany) and from the German Federal Ministry of Education and Research (BMBF) to the German Center for Diabetes Research (DZD e.V.).

\section{Competing interests None declared}

Patient consent Not required.

Ethics approval The GDS was approved by the ethics committee of Heinrich Heine University Düsseldorf (study reference number 4508, previous reference number 2478).

Provenance and peer review Not commissioned; externally peer reviewed.

Data sharing statement All available data can be obtained from the corresponding author.

Open access This is an open access article distributed in accordance with the Creative Commons Attribution Non Commercial (CC BY-NC 4.0) license, which permits others to distribute, remix, adapt, build upon this work non-commercially, and license their derivative works on different terms, provided the original work is properly cited, appropriate credit is given, any changes made indicated, and the use is non-commercial. See: http://creativecommons.org/licenses/by-nc/4.0/.

\section{REFERENCES}

1. Al Sayah F, Majumdar SR, Williams B, et al. Health literacy and health outcomes in diabetes: a systematic review. J Gen Intern Med 2013;28:444-52.

2. Bunge M, Mühlhauser I, Steckelberg A. What constitutes evidencebased patient information? Overview of discussed criteria. Patient Educ Couns 2010;78:316-28.

3. Duggan C, Bates I. Medicine information needs of patients: the relationships between information needs, diagnosis and disease. Qual Saf Health Care 2008;17:85-9.

4. Biernatzki L, Kuske S, Genz J, et al. Information needs in people with diabetes mellitus: a systematic review. Syst Rev 2018;7:27.

5. Wilkinson E, Randhawa G, Singh M. Quality improvements in diabetes care, how holistic have they been? A case-study from the United Kingdom. Int J Equity Health 2014;13:29.

6. Ormandy P. Defining information need in health - assimilating complex theories derived from information science. Health Expect 2011;14:92-104.

7. Szendroedi J, Saxena A, Weber KS, et al. Cohort profile: the German Diabetes Study (GDS). Cardiovasc Diabetol 2016;15:59.

8. Chernyak N, Stephan A, Bächle C, et al. Assessment of information needs in diabetes: Development and evaluation of a questionnaire. Prim Care Diabetes 2016;10:287-92.

9. Leech NL, Onwuegbuzie AJ. A typology of mixed methods research designs. Qual Quant 2009;43:265-75.

10. Mühlhauser I, Sawicki P, Didjurgeit U, et al. Uncontrolled hypertension in type 1 diabetes: assessment of patients' desires about treatment and improvement of blood pressure control by a structured treatment and teaching programme. Diabet Med 1988;5:693-8.

11. Ankem K. Factors influencing information needs among cancer patients: a meta-analysis. Libr Inf Sci Res 2006;28:7-23.

12. St Jean B. Information behavior of people diagnosed with a chronic serious health condition: a longitudinal study. Ann Arbor: The University of Michigan, 2012.
13. van Esch SC, Cornel MC, Snoek FJ. "I am pregnant and my husband has diabetes. Is there a risk for my child?" A qualitative study of questions asked by email about the role of genetic susceptibility to diabetes. BMC Public Health 2010;10:688.

14. Lamberts EJ, Bouvy ML, van Hulten RP. The role of the community pharmacist in fulfilling information needs of patients starting oral antidiabetics. Res Social Adm Pharm 2010;6:354-64.

15. Wilson V. Patient use of the internet for diabetes information. Nurs Times 2013;109:18-20.

16. Kuske S, Schiereck T, Grobosch S, et al. Diabetes-related information-seeking behaviour: a systematic review. Syst Rev 2017;6:212.

17. Degner LF, Sloan JA, Venkatesh P. The control preferences scale. Can J Nurs Res 1997;29:21-43.

18. Schram MT, Baan CA, Pouwer F. Depression and quality of life in patients with diabetes: a systematic review from the European depression in diabetes (EDID) research consortium. Curr Diabetes Rev 2009:5:112-9.

19. Hasan SS, Mamun AA, Clavarino AM, et al. Incidence and risk of depression associated with diabetes in adults: evidence from longitudinal studies. Community Ment Health J 2015;51:204-10.

20. Hautzinger M, Bailer M, Hofmeister D, et al. Allgemeine Depressionsskala (ADS). Göttingen: Hogrefe Verlag, 2012.

21. Polonsky WH, Anderson BJ, Lohrer PA, et al. Assessment of diabetes-related distress. Diabetes Care 1995;18:754-60.

22. Bengel J, Wirtz M, Zwingmann C. Diagnostische Verfahren in der Rehabillitation. Göttingen: Hogrefe Verlag, 2008.

23. Ware JE, Kosinski M, Keller SD. SF-36 physical and mental health summary scales: a user's manual. Boston, MA: Health Assessment Lab, 1994.

24. Bullinger M, Kirchberger I, Ware J. The German SF-36 health survey translation and psychometric testing of a generic instrument for the assessment of health-related quality of life. Journal of Public Health 1995;3:21.

25. Brähler E, Mühlan $\mathrm{H}$, Albani $\mathrm{C}$, et al. Teststatistische Prüfung und Normierung der deutschen Versionen des EUROHIS-QOL Lebensqualität-Index und des WHO-5 Wohlbefindens-Index. Diagnostica 2007;53:83-96.

26. WHO Global NCD Infobase Team \& World Health Organization Dept. of Noncommunicable Disease Surveillance. Surveillance of chronic disease risk factors: country-level data and comparable estimates. Geneva: World Health Organization, 2005.

27. Baecke JA, Burema J, Frijters JE. A short questionnaire for the measurement of habitual physical activity in epidemiological studies. Am J Clin Nutr 1982;36:936-42.

28. Florindo AA, Latorre MdoRDdeO. Validation and reliability of the Baecke questionnaire for the evaluation of habitual physica activity in adult men. Revista Brasileira de Medicina do Esporte 2003;9:129-35.

29. Elo S, Kyngäs $\mathrm{H}$. The qualitative content analysis process. $J$ Adv Nurs 2008;62:107-15.

30. Hernán MA, Hernández-Díaz S, Robins JM. A structural approach to selection bias. Epidemiology 2004;15:615-25.

31. St. Jean B. Factors motivating, demotivating, or impeding information seeking and use by people with type 2 diabetes: a call to work toward preventing, identifying, and addressing incognizance. $J$ Assoc Inf Sci Technol 2017;68:309-20.

32. Beeney LJ, Bakry AA, Dunn SM. Patient psychological and information needs when the diagnosis is diabetes. Patient Educ Couns 1996;29:109-16.

33. Hjelm K, Berntorp K, Frid A, et al. Beliefs about health and illness in women managed for gestational diabetes in two organisations. Midwifery 2008;24:168-82.

34. St. Jean B. Devising and implementing a card-sorting technique for a longitudinal investigation of the information behavior of people with type 2 diabetes. Libr Inf Sci Res 2014;36:16-26.

35. Strandberg RB, Graue M, Wentzel-Larsen T, et al. The relationships among fear of hypoglycaemia, diabetes-related quality of life and psychological well-being in Norwegian adults with Type 1 diabetes. Diabetes Res Clin Pract 2017;124:11-19.

36. Horigan G, Davies M, Findlay-White F, et al. Reasons why patients referred to diabetes education programmes choose not to attend: a systematic review. Diabet Med 2017;34:14-26.

37. Hartwig S, Kluttig A, Tiller D, et al. Anthropometric markers and their association with incident type 2 diabetes mellitus: which marker is best for prediction? Pooled analysis of four German populationbased cohort studies and comparison with a nationwide cohort study. BMJ Open 2016;6:e009266. 